\title{
Edge-disjoint branchings in temporal digraphs
}

\author{
Victor Campos* Raul Lopes* \\ Departamento de Computação \\ Universidade Federal do Ceará \\ Fortaleza, CE, Brazil \\ victoitor@ufc.br; raul@alu.ufc.br
}

\author{
Andrea Marino \\ Ana Silva \\ Dipartimento di Sistemi, Informatica, Applicazioni \\ Università degli Studi di Firenze \\ Firenze, Italy \\ Departamento de Matemática \\ Universidade Federal do Ceará \\ Fortaleza, CE, Brazil \\ andrea.marino@unifi.it \\ anasilva@mat.ufc.br
}

Submitted: Feb 5, 2021; Accepted: Sep 10, 2021; Published: Oct 8, 2021

(C) The authors. Released under the CC BY-ND license (International 4.0).

\begin{abstract}
A temporal digraph $\mathcal{G}$ is a triple $(G, \gamma, \lambda)$ where $G$ is a digraph, $\gamma$ is a function on $V(G)$ that tells us the time stamps when a vertex is active, and $\lambda$ is a function on $E(G)$ that tells for each $u v \in E(G)$ when $u$ and $v$ are linked. Given a static digraph $G$, and a subset $R \subseteq V(G)$, a spanning branching with root $R$ is a subdigraph of $G$ that has exactly one path from $R$ to each $v \in V(G)$. In this paper, we consider the temporal version of Edmonds' classical result about the problem of finding $k$ edgedisjoint spanning branchings respectively rooted in given $R_{1}, \cdots, R_{k}$. We introduce and investigate different definitions of spanning branchings, and of edge-disjointness in the context of temporal digraphs. A branching $\mathcal{B}$ is vertex-spanning if the root is able to reach each vertex $v$ of $G$ at some time where $v$ is active, while it is temporal-spanning if each $v$ can be reached from the root at every time where $v$ is active. On the other hand, two branchings $\mathcal{B}_{1}$ and $\mathcal{B}_{2}$ are edge-disjoint if they do not use the same edge of $G$, and are temporal-edge-disjoint if they can use the same edge of $G$ but at different times. This lead us to four definitions of disjoint spanning branchings and we prove that, unlike the static case, only one of these can be computed in polynomial time, namely the temporal-edge-disjoint temporalspanning branchings problem, while the other versions are NP-complete, even under very strict assumptions.
\end{abstract}

Mathematics Subject Classifications: 05C85, 68Q25

*Partially supported by FUNCAP/CNPq/Brazil, Project PRONEM PNE-0112-00061.01.00/16, CNPq Produtividade 303803/2020-7 and 313642/2018-4 


\section{Introduction}

A temporal digraph is a digraph that changes in time. That is, given a static digraph $G$, a temporal digraph $\mathcal{G}$ with base static digraph $G$ and lifetime set $\mathcal{T}$ changes as follows: at each time stamp $t \in \mathcal{T}$, only a subdigraph of $G$ is active, and edges might have a delay, leaving a vertex at some time stamp but arriving only later. The maximum value in $\mathcal{T}$ is called the lifetime of $\mathcal{G}$. If a vertex $v \in V(G)$ is active at every $t \in \mathcal{T}$, we say that $v$ is permanent. We will refer to static digraphs simply as digraphs and, when referring to temporal digraphs, we will always use the complete term. We restrict our attention to digraphs that may have multiple edges and $\mathcal{T} \subseteq \mathbb{N}$.

In this paper we deal with disjoint spanning branchings in temporal digraphs, which are well-understood structures in digraphs due to a characterization by Edmonds [10]. In this paper, we work on a formulation due to Schrijver [25]. Given a digraph $G$, and a subset $R \subseteq V(G)$, we say that $H \subseteq G$ is a spanning branching of $G$ with root $R$ if $V(H)=V(G)$, and $H$ contains exactly one path between some $r \in R$ and $u$, for each $u \in V(G)$. Edmonds' result [10] gives a necessary and sufficient condition for the existence of $k$ edge-disjoint branchings given root sets $R_{1}, \ldots, R_{k}$. A polynomial time algorithm for finding these branchings can be obtained from Lovász [18] and Tarjan [28].

When translating concepts to temporal (di)graphs, it is often the case that theorems coming from graph theory, in the classical sense, can hold or not depending on the adopted definition. Indeed, in [16] the authors give an example where Edmonds' result on branchings does not hold on the temporal context. However, as we will see later, their concept is just one of many possible definitions, which can behave very differently in terms of complexity.

Another example of such behavior is the validity of Menger's Theorem. It has been shown that the edge version of Menger's Theorem holds [2], even if one considers weights on the edges [1]. However, the vertex version of Menger's Theorem holds or not, depending on how one interprets what a cut should be $[2,16,20]$.

Our contribution. Given a temporal digraph $\mathcal{G}$ with base digraph $G$, and subsets of vertices in time $R_{1}, \cdots, R_{k}$, i.e., sets of pairs $(u, t)$ where $u$ is a vertex of $G$ and $t$ a time stamp, here we investigate the many variations of finding (pairwise) disjoint spanning branchings with roots $R_{1}, \cdots, R_{k}$. Spanning can mean that one wants to pass by at least one appearance of each $u \in V(G)$ (called vertex-spanning), or by all appearances of each $u \in V(G)$ (called temporal-spanning). Similarly, edge-disjoint can have different interpretations, as it can refer to edges of $G$ or to the appearances of these edges in $\mathcal{G}$. We say that two branchings are edge-disjoint if they do not share any edge of $G$, and that they are temporal-edge-disjoint (or t-edge-disjoint for short) if they do not share any appearance of an edge of $G$ in $\mathcal{G}$.

We found that the only case in which this problem is polynomial (as its static counterpart) is when we want $t$-edge-disjoint temporal-spanning branchings. In addition, whenever edges have a delay, i.e. they require a traversal time, the latter problem becomes linear on the size of the temporal graph. This is because we have found that, given a directed acyclic static graph (in short, DAG) $D$, finding $k$ edge-disjoint spanning branch- 
ings in $D$ can be done in linear time, if they exist. On the other hand, having delay on the edges does not make the other cases easier, since our NP-completeness proofs extend to them.

We also have found that if vertices are permanent (this is the more popular case where vertices are always active), the problem is polynomial for temporal-spanning branchings and NP-complete otherwise.

Our results are summarized in Table 1 and detailed in the following main theorem. A digraph $G$ is an in-star if there exists $u \in V(G)$ such that all the edges in $G$ are incoming edges to $u$.

Theorem 1. Let $\mathcal{G}$ be a temporal digraph with base digraph $G, k \geqslant 2$ be a fixed integer, and consider subsets of vertices in time, $R_{1}, \cdots, R_{k}$. The problem of finding $k$ branchings rooted in $R_{1}, \cdots, R_{k}$ is:

1. Polynomial for t-edge-disjoint temporal-spanning, and linear on the size of $\mathcal{G}$ if all edges have a delay or if each snapshot is a DAG.

2. NP-complete for edge-disjoint temporal-spanning even if $G$ is an in-star, and each snapshot has constant size, or if $\mathcal{G}$ has lifetime 3. Furthermore, if vertices are permanent or $\mathcal{G}$ has lifetime 2 , then edge-disjoint temporal-spanning can be solved in polynomial time, and in linear time on the size of $\mathcal{G}$ if all edges have a delay or if each snapshot is a $D A G$.

3. NP-complete for edge-disjoint vertex-spanning even if $G$ is a $D A G$, the lifetime of $\mathcal{G}$ is 2 , and vertices are permanent.

4. NP-complete for $t$-edge-disjoint vertex-spanning even if $G$ is a $D A G$, the lifetime of $\mathcal{G}$ is 2 , and vertices are permanent.

Furthermore, the NP-completeness results still hold even if either all or none of the temporal edges have delay, with the lifetime in Items 3 and 4 increasing to 5.

Finally, our reductions further imply that, in the case of edge-disjoint temporalspanning, even if the base digraph $G$ is an in-star, the problem cannot be solved by an algorithm running in time $O^{*}\left(2^{o(T)}\right)$ unless ETH fails, where $T$ is the lifetime of $\mathcal{G}$. Moreover, in the vertex-spanning variations, the problem also cannot be solved in $O^{*}\left(2^{o(n+m)}\right)$ under the same assumption, where $n$ and $m$ are respectively the number of vertices and edges of the base digraph of $\mathcal{G}$.

A preliminary version of our work appeared in [7].

Related Work: While it is easy to imagine a variety of graph problems that can profit from considering changes in time, it is hard to pin-point when the study of temporal graphs and similar structures began. Nevertheless, in the last decade or so, it has attracted a lot of attention from the community, with a considerable number of papers being published in the field (we refer the reader to the surveys $[17,21]$ ). We mention that temporal graphs (or other very similar structures) appear in the literature under a number of names, 


\begin{tabular}{|c|c|c|c|c|}
\cline { 2 - 5 } \multicolumn{1}{c|}{} & \multicolumn{2}{c|}{ NOT PERMANENT VERTICES } & \multicolumn{2}{c|}{ PERMANENT VERTICES } \\
\cline { 2 - 5 } \multicolumn{1}{c|}{} & EDGE-DISJOINT & T-EDGE-DISJOINT & EDGE-DISJOINT & T-EDGE-DISJOINT \\
\hline $\begin{array}{c}\text { TEMPORAL- } \\
\text { SPANNING }\end{array}$ & NP-c & Poly* & Poly* & Poly* \\
\hline $\begin{array}{c}\text { VERTEX- } \\
\text { SPANNING }\end{array}$ & NP-c & NP-c & NP-c & NP-c \\
\hline
\end{tabular}

Table 1: Our results. Vertices are permanent if they are always active. Starred cells mean that the problem becomes linear whenever the temporal edges have delay or each snapshot is a DAG.

such as dynamic networks [3], time-varying graphs [8], evolving networks [5], and link streams [17]. Also, many works consider a temporal graph $\mathcal{G}$ as having vertices that are always active, and edges have the same starting and ending time [1,6,16,20,23]. While models where edges that have a delay are more common $[8,30]$, models where nodes can be inactive have also been considered in $[8,17]$.

A path in temporal graphs is generally understood as a sequence of edges respecting time, i.e. the arrival time in each internal vertex of the path cannot be higher than the departing time of the next edge leaving this vertex. In this context, a number of metrics can be related to a path, such as earliest arrival time, latest departure time, minimum number of temporal edges, and minimum traveling time [30]. When vertices can be inactive, we have to further ensure that, when waiting for the next edge on a certain vertex, it must remain active in the waiting period [8]. In this scenario, the definitions of reachability and connectivity change accordingly, and it is natural to ask how well-known structures and results from graph theory in the classical sense change taking into account the temporal constraint.

We mention that most of the applications seem to use only t-edge-disjoint paths between vertices of the underlying graph, i.e. leaving out Items (1), (2), and (3) of Theorem 1. However, as we already mentioned, one of the challenges encountered in the theory of temporal graphs is facing with different possible definitions. These possibilities depend on the treatment of vertices and edges of the underlying graph in relation to the treatment of their temporal occurrences. For instance, one of the most important theorems in graph theory is Menger's Theorem, which says that, given a graph $G$ and vertices $u, v \in V(G)$, the maximum number of internally disjoint $u, v$-paths equals the minimum size of a $u, v$-cut (set of vertices whose removal disconnect $u$ and $v$ ). Observe that, using a similar reasoning as the one adopted here, we could talk about many different versions of Menger's Theorem, depending on whether the disjointness of the paths considers temporal vertices or regular ones (let us call these t-vertex disjoint and vertex-disjoint, respectively), and whether a cut is a subset of vertices or a subset of temporal vertices (let us call these $u, v$-cut and $u, v$-t-cut, respectively). In [2] and [16] they give simple examples where Menger's Theorem does not work on the temporal context. Their examples fall in the category vertex-disjoint, $u, v$-cut, i.e., the number of vertex-disjoint temporal walks between a pair of vertices might be smaller than the size of a $u, v$-cut. Figure 1 shows the 
example presented in [16]. In contrast to this result, in [20] they give a valid formulation for a temporal version of Menger's Theorem by introducing a new cut concept. Without going into details, we mention that they actually ask that two temporal walks do not share the same departure time of any vertex (let us call this departure-time-disjoint temporal walks), and prove that the maximum number of departure-time-disjoint temporal walks from a vertex $u$ to a vertex $v$ is equal to the mininum size of a departure-time $u, v$-cut. Hence, different conclusions can be (and have been) made about the temporal version of Menger's Theorem depending on the adopted translation in terms of temporal graphs.

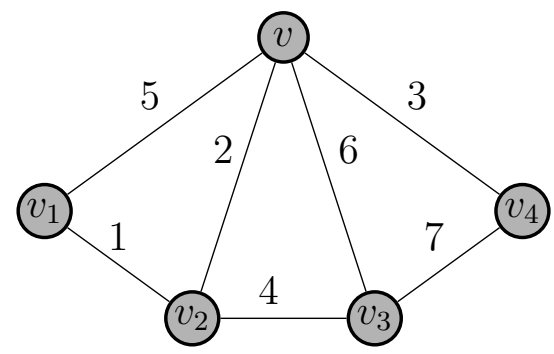

Figure 1: Example presented in [16]. The only way to disconnect $v_{1}$ from $v_{4}$ is to remove at least two vertices between $\left\{v, v_{2}, v_{3}\right\}$, while there are no internally vertex-disjoint temporal walks between $v_{1}$ and $v_{4}$.

Temporal definitions of trees [6,17] and (minimum) spanning trees [15], which are related to our definition of branching, have been proposed and investigated, and usually consist of ensuring that the root-to-node path in the tree is a valid temporal path. Analogously, temporal cuts from a vertex $s$ to $t$ aim to break any temporal path from $s$ to $t$ and can be related to extending the max-flow min-cut Theorem to temporal digraphs [1]. Edmonds' Theorem on disjoint branchings is a classical theorem in graph theory, with many distinct existing proofs (e.g. Lovász [18], Tarjan [28], and Fulkerson and Harding [14]), and has many interesting consequences on digraph theory (e.g., one can derive Menger's Theorem from it, characterize arc-connectivity [26], characterize branching cover [13], ensure integer decomposition of the polytope of branchings of size $k$ [19], etc). As far as we know, the only other time that Edmonds' Theorem has been investigated on the temporal context has been in [16], where the authors give an example where the theorem does not hold. The definition used by them falls into our category of edge-disjoint vertex-spanning branchings, which we prove to be NP-complete even under very strict constraints.

Structure of the paper. The paper is organized as follows. In Section 2, we formalize the definitions of spanning branchings and disjointness, also showing that having multiple roots in each of the $k$ branchings is computationally equivalent to having a single root for all of the $k$ branchings. In Section 3, we present the results about temporal-spanning branchings. In Section 4 we present our results concerning vertex-spanning branchings. In Section 5, we discuss some possible formulations of Edmonds' Condition in the temporal context and why we do not believe such a condition can even exist. Finally, in Section 6, we draw our conclusions and make some final remarks. 


\section{Terminology and preliminary results}

A digraph $G$ is a triple consisting of a vertex set $V(G)$, an edge set $E(G)$ and a function assigning each edge to an ordered pair of vertices. The first vertex of the ordered pair is the tail of an edge, and the second is the head; together, they are the endpoints. We say that an edge goes from its tail to its head. Multiple edges are edges having the same ordered pair of endpoints. We denote an edge $e$ from $u$ to $v$ by $u v$ when it does not cause any confusion with other edges on the same endpoints. Other than this slight abuse of notation, we use standard graph theoretic notation which can be found on the textbooks of West [29] or Bondy and Murty [4].

Given vertices $v_{0}, v_{k}$ in a digraph $G$, a $v_{0}, v_{k}$-walk in $G$ is an alternating sequence $\left(v_{0}, e_{1}, v_{1}, \ldots, e_{k}, v_{k}\right)$ of alternating vertices and edges such that $e_{i}$ goes from $v_{i-1}$ to $v_{i}$ for $i \in\{1, \ldots, k\}$. We say that a $u, v$-walk is a walk from $u$ to $v$. If such a walk exists, we say that $u$ reaches $v$. Given a subset $R \subseteq V(G)$, we say that $H \subseteq G$ is a spanning branching of $G$ with root $R$ if $V(H)=V(G)$, and $H$ contains exactly one walk from some $r \in R$ to $u$, for each $u \in V(G)$. Edmonds [10] characterized when there exist $k$ edge disjoint branchings in a digraph and a polynomial time algorithm for finding these branchings can be obtained from Lovászz [18] and Tarjan [28]. In this paper, we will cite Edmonds' Theorem to mean that such branchings can be found in polynomial time.

Although Graph Theory is close to 300 years old, it is interesting to see how notation has evolved even from 50 years ago to today. This leads to an important trend to move into a standard notation among recent textbooks. Given that the study of temporal graphs is relatively young, we note that different papers have vastly different notation. One of the reasons behind these differences is the context of the application, which often implies different assumptions on how the input is given. This is the case for instance of time-varying graphs [23], temporal graphs [21], and link streams [17].

In this paper, we use the notation in [21] and extend it in a very natural way to include the case in which not only the edges, but also the vertices can become inactive. This allows us to state our results in a setting which is as general as possible. For the sake of description, we also give names to notions and concepts recurrent in our paper.

A temporal digraph $\mathcal{G}$ is a triple $(G, \gamma, \lambda)$ where $G$ is a digraph and $\gamma$ and $\lambda$ are functions on $V(G)$ and $E(G)$, respectively, that tell us when the vertices and the edges appear. More formally, for each $v \in V(G)$ we have $\gamma(v) \subseteq \mathbb{N}^{*}$, and for each edge $e \in E(G)$ we have $\lambda(e) \subseteq \mathbb{N}^{*} \times \mathbb{N}^{*}$ where $\mathbb{N}^{*}$ is the set of positive natural numbers. Furthermore, if $\left(t, t^{\prime}\right) \in \lambda(u v)$, then $t \in \gamma(u), t^{\prime} \in \gamma(v)$, and $t \leqslant t^{\prime}$. We call $G$ the base digraph, $T(\mathcal{G})=\max \bigcup_{v \in V(G)} \gamma(v)$ the lifetime and $\mathcal{T}(\mathcal{G})=\{1, \ldots, T(\mathcal{G})\}$ the lifetime set of $\mathcal{G}$. In this paper, we consider only finite temporal digraphs where $G$ and $\mathcal{T}(\mathcal{G})$ are finite.

The vertices and edges of $\mathcal{G}$ are the vertices and edges of $G$. We say that a vertex $v$ is active at time $t$ if $t \in \gamma(v)$, and that $v$ is active from $t_{1}$ to $t_{2}$ if $v$ is active for every time $t$ with $t_{1} \leqslant t \leqslant t_{2}$. Moreover, we say that $v$ is permanent if $v$ is active throughout the lifetime of $\mathcal{G}$, i.e., $\gamma(v)=\mathcal{T}(\mathcal{G})$. For $e \in E(G)$, we say that $e$ has a delay if $t \neq t^{\prime}$ for every $\left(t, t^{\prime}\right) \in \gamma(e)$ and we say that $e$ is instantaneous if $t=t^{\prime}$ for every $\left(t, t^{\prime}\right) \in \gamma(e)$. The set $V_{T}(\mathcal{G})$ of temporal vertices is the set $\{(v, t) \mid v \in V(G)$ and $t \in \gamma(v)\}$, and the set $E_{T}(\mathcal{G})$ 
of temporal edges is the set $\left\{\left(e, t, t^{\prime}\right) \mid e \in E(G)\right.$ and $\left.\left(t, t^{\prime}\right) \in \lambda(e)\right\}$; if $\mathcal{G}$ is clear from the context, we omit it from the notation. Observe that a temporal digraph $\mathcal{G}=(G, \gamma, \lambda)$ can be also seen as a pair of digraphs $\left(G, G_{T}\right)$ where $V\left(G_{T}\right)=V_{T}, E\left(G_{T}\right)=E_{T}$ and $\left(e, t, t^{\prime}\right) \in E_{T}$ goes from $(u, t)$ to $\left(v, t^{\prime}\right)$ when $e=u v$. When $t=t^{\prime}$, we may denote the temporal edge $\left(e, t, t^{\prime}\right)$ simply by $(e, t)$. This is similar to what has been proposed in [1] and [8]. We call the digraph $G_{T}$ the $(\gamma, \lambda)$-digraph of $\mathcal{G}$. Also, if not clear from the context, we use $V_{T}(\mathcal{G})$ and $E_{T}(\mathcal{G})$ to denote the temporal vertex set and the temporal edge set of $\mathcal{G}$, respectively.

We say that a temporal graph is permanent if every vertex is permanent and we say that it is instantaneous if every edge is instantaneous. We note that the definition of temporal graphs given in [16] corresponds to our definition of a permanent and instantaneous temporal graph. Our definitions also generalize the definitions of stream graph given in [17], and of time-varying graphs given in [8].

When presenting an instantaneous temporal digraph $\mathcal{G}=(G, \gamma, \lambda)$, we may present, for each timestamp, the active subgraph of $G$. For $i \in \mathcal{T}(\mathcal{G})$, the subgraph of $G$ with vertex set $\{u \in V(G) \mid i \in \gamma(u)\}$ and edge set $\{e \in E(G) \mid(i, i) \in \lambda(e)\}$ is called the $i$-snapshot of $\mathcal{G}$ and is denoted by $G_{i}$. A subgraph of $G$ is called a snapshot if it is the $i$-snapshot for some $i \in \mathcal{T}(\mathcal{G})$.

A temporal walk is the analogous concept to a walk in a digraph. Given temporal vertices $s_{1}, s_{k} \in V_{T}$, an $s_{1}, s_{k}$-temporal walk in $\left(G, G_{T}\right)$ is a sequence of temporal vertices and temporal edges, $s_{1}, \ldots, s_{k}$, such that: if $s_{i}$ is a temporal edge, then $s_{i-1}$ and $s_{i+1}$ are temporal vertices and $s_{i}$ goes from $s_{i-1}$ to $s_{i+1}$; and if $s_{i}$ and $s_{i+1}$ are temporal vertices, then $s_{i}=(v, t)$ and $s_{i+1}=(v, t+1)$ for some vertex $v$ and some time $t$. We say that an $s_{1}, s_{k}$-temporal walk is a temporal walk from $s_{1}$ to $s_{k}$. If such a walk exists, we say that $s_{1}$ reaches $s_{k}$. See Figure 2 for an example.

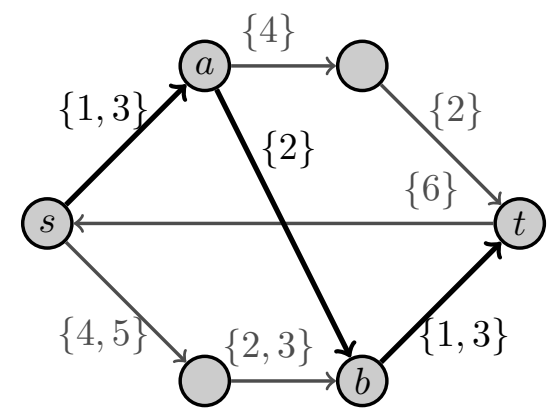

Figure 2: Example of a temporal walk in a temporal digraph which is permanent and instantaneous. Next to each edge we depict the snapshots where that edge is active. In the figure, the sequence $((s, 1),(s a, 1),(a, 1),(a, 2),(a b, 2),(b, 2),(b, 3),(b t, 3),(t, 3))$ is a temporal walk from $(s, 1)$ to $(t, 3)$.

For temporal digraphs $\mathcal{B}=\left(G^{\prime}, \gamma^{\prime}, \lambda^{\prime}\right)$ and $\mathcal{G}=(G, \gamma, \lambda)$, we say that $\mathcal{B}$ is a temporal subdigraph of $\mathcal{G}$ if $G^{\prime} \subseteq G, \gamma^{\prime}(v) \subseteq \gamma(v)$ for $v \in V\left(G^{\prime}\right)$ and $\lambda^{\prime}(e) \subseteq \lambda(e)$ for $e \in E\left(G^{\prime}\right)$. Let $R \subseteq V_{T}(\mathcal{G})$; a temporal subdigraph $\mathcal{B}$ of $\mathcal{G}$ is a temporal-spanning branching of $\mathcal{G}$ with 
root $R$ if, for every $(v, j) \in V_{T}(\mathcal{G})$, there exists $(u, i) \in R$ such that $(u, i)$ reaches $(v, j)$ in $\mathcal{B}$; and we say $\mathcal{B}$ is a vertex-spanning branching of $\mathcal{G}$ with root $R$ if, for every $v$ in $V(G)$, there exists $(u, i) \in R$ and $(v, j) \in V_{T}$ such that $(u, i)$ reaches $(v, j)$ in $\mathcal{B}$.

Note that the definition of branchings in digraphs requires that a unique walk from the root set to each vertex is taken. In other words, one is interested in a minimal subdigraph such that all vertices can be reached from the root. Observe that if we take a subdigraph with the reachability property, but not necessarily minimal, one can obtain a minimal one simply by discarding any unnecessary edges. In the temporal context, we drop the minimality requirement and consider walks instead of paths for a number of reasons. First, we believe that the main desired property of a spanning branching should be reachability and not uniqueness of reachability. Second, the uniqueness of a walk cannot be insured in a vertex-spanning branching, as can be seen in Figure 3. Finally, minimality is irrelevant when looking for disjoint temporal branchings as a minimal representation can be obtained in polynomial time, not affecting the complexity of the problem.
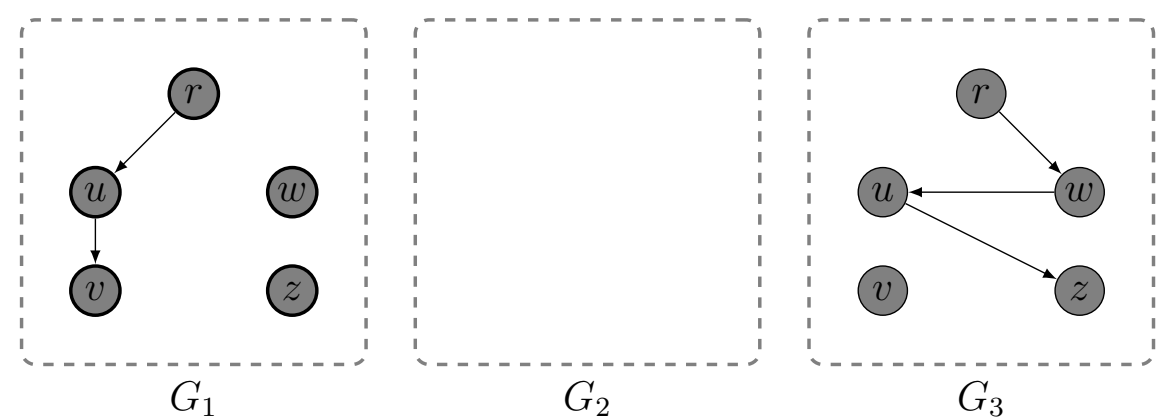

Figure 3: Example of a temporal digraph that contains a vertex-spanning branching that needs to pass by the same vertex twice, namely, vertex $u$.

Given two branchings $\mathcal{B}_{1}=\left(G^{\prime}, \gamma_{1}, \lambda_{1}\right)$ and $\mathcal{B}_{2}=\left(G^{\prime \prime}, \gamma_{2}, \lambda_{2}\right)$ rooted in $R_{1}, R_{2}$, respectively, either both temporal-spanning or both vertex-spanning, we say that $\mathcal{B}_{1}$ and $\mathcal{B}_{2}$ are temporal-edge-disjoint (or t-edge-disjoint for short) if they have no common temporal edges; more formally, if $\lambda_{1}(e) \cap \lambda_{2}(e)=\emptyset$ for every $e \in E\left(G^{\prime}\right) \cap E\left(G^{\prime \prime}\right)$. And we say that $\mathcal{B}_{1}$ and $\mathcal{B}_{2}$ are edge-disjoint if there is no edge in $E(G)$ that has copies in both $\mathcal{B}_{1}$ and $\mathcal{B}_{2}$; more formally, if $E\left(G^{\prime}\right) \cap E\left(G^{\prime \prime}\right)=\emptyset$.

Problem 2 ( $\boldsymbol{k} \boldsymbol{X}$-disjoint $\boldsymbol{Y}$-spanning Branching). Let $X \in$ \{edge,t-edge\}, $Y \in$ $\{$ temporal, vertex $\}$, and $k$ be a fixed positive integer. Given a temporal digraph $\mathcal{G}$, and subsets of temporal vertices $R_{1}, \ldots, R_{k} \subseteq V_{T}$, find $k X$-disjoint $Y$-spanning branchings $\mathcal{B}_{1}, \ldots, \mathcal{B}_{k}$ respectively with $\operatorname{roots} R_{1}, \ldots, R_{k}$.

We introduce the following restriction of Problem 2, which corresponds to finding branchings that have a single root (also called out-arborescence).

Problem 3 ( $\boldsymbol{k}$ Single Source $\boldsymbol{X}$-disjoint $\boldsymbol{Y}$-spanning Branching). Let $X \in$ \{edge, t-edge , $Y \in\{$ temporal, vertex $\}$, and $k$ be a fixed positive integer. Given a temporal digraph $\mathcal{G}$, 
and a temporal vertex $r \in V_{T}$, find $k X$-disjoint $Y$-spanning branchings $\mathcal{B}_{1}, \ldots, \mathcal{B}_{k}$ each one with root $r$.

Lemma 4. Problem 2 is computationally equivalent to Problem 3.

Proof. Problem 3 is clearly a restriction of Problem 2. In the following we provide the reduction in the opposite direction, from the problem where each branching has a subset of $V_{T}$ as roots to the problem where each branching has a single same root. So let $\left(\mathcal{G}, R_{1}, \ldots, R_{k}\right)$ be an instance of Problem 2 . We suppose without, loss of generality, that no vertices are active at time 1 ; for this it suffices to add one to the labels in $\gamma, \lambda$. For each $i \in\{1, \ldots, k\}$ add a new vertex $r_{i}$ to $G$ and the edge $r_{i} u$ for every $u \in V(G)$ such that $(u, t) \in R_{i}$. Then, make $\gamma\left(r_{i}\right)=\{1\}$, and for each $(u, t) \in R_{i}$, add $(1, t)$ to $\lambda\left(r_{i} u\right)$ (which is the same as adding the temporal edge $\left(r_{i} u, 1, t\right)$ to $\mathcal{G}$ ). Moreover, add a vertex $r$ and the edge $r r_{i}$ for $i \in\{1, \ldots, k\}$; also make $\gamma(r)=\{1\}$ and $\lambda\left(r r_{i}\right)=\{(1,1)\}$ (which is the same as adding temporal edges $\left(r r_{i}, 1,1\right)$ for every $\left.i \in\{1, \cdots, k\}\right)$.

One can see that $k$ vertex-spanning (resp. temporal-spanning) branchings rooted in $r$ give $k$ vertex-spanning (resp. temporal-spanning) branchings rooted in $R_{1}, \cdots, R_{k}$, and vice-versa. The edge-disjointness, both for t-edge or edge-disjoint versions, clearly are not altered by adding the new temporal edges.

The next easy proposition tells us that finding $k$ disjoint spanning branchings is hard, for some fixed $k$, then so is finding $k+1$ of them.

Proposition 5. Let $X \in\{$ edge,t-edge $\}, Y \in\{$ temporal, vertex $\}$ and $k$ be a fixed positive integer. If Problem $k X$-disjoint $Y$-spanning Branching is NP-complete, then the same holds for Problem $k+1 X$-disjoint $Y$-spanning Branching.

Proof. To reduce from $k$ to $k+1$, it suffices to add $R_{k+1}=V_{T}$ as an entry. Surely the $(k+1)$-th branching can be chosen with no temporal edges, which means that the others form a solution to the initial problem.

\section{Temporal-Spanning Branchings}

This section is devoted to study Problem 2 in the case where $Y$ is temporal, i.e. we aim to find $k X$-disjoint temporal-spanning branchings, with $X \in$ \{edge,t-edge $\}$. This corresponds to items 1 and 2 from Theorem 1.

\subsection{T-edge-disjoint Temporal-Spanning Branchings}

Let $\mathcal{G}=(G, \gamma, \lambda)$ and $R_{1}, \cdots, R_{k} \subseteq V_{T}$. Let $H_{k}$ be a digraph obtained from $G_{T}$ by adding $k$ parallel edges from $(u, t)$ to $(u, t+1)$ whenever $\{(u, t),(u, t+1)\} \subseteq V_{T}$. See Figure 4 for an example.

We prove that $\mathcal{G}$ has the desired branchings if and only if $H_{k}$ has $k$ edge-disjoint spanning branchings with roots $R_{1}, \cdots, R_{k}$. Then, the first part of Item 1 of Theorem 1 follows by Edmonds' Theorem [10]. The second part is proven in Section 3.1.1. 

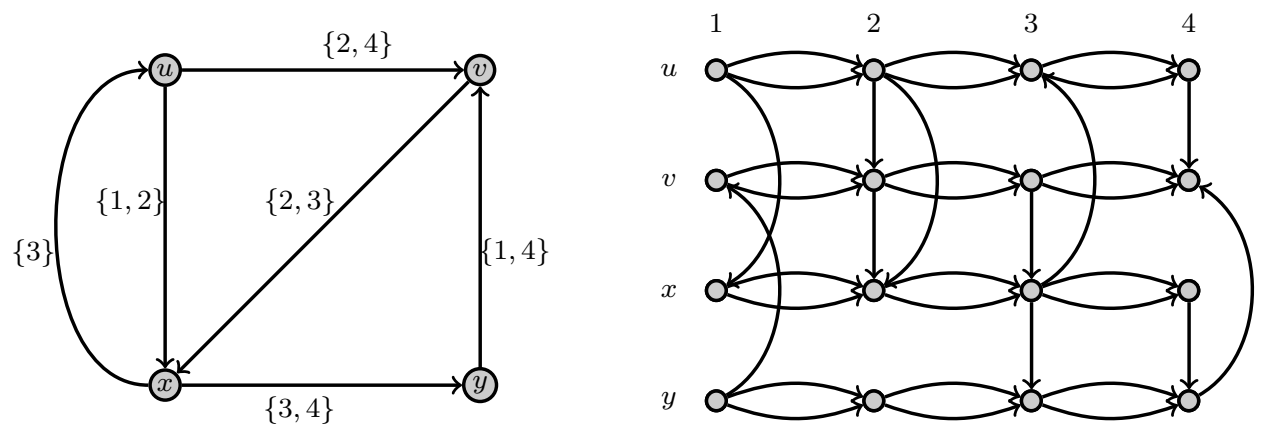

Figure 4: On the right, the digraph $H_{2}$ related to the temporal digraph depicted to the left. For simplicity, we consider only permanent vertices and instantaneous edges.

Lemma 6. Let $\mathcal{G}=(G, \gamma, \lambda)$ be a temporal digraph, $R_{1}, \cdots, R_{k} \subseteq V_{T}$, and $H_{k}$ be constructed as above. Then, $\mathcal{G}$ has $k$ t-edge-disjoint temporal-spanning branchings rooted in $R_{1}, \cdots, R_{k}$ if and only if $H_{k}$ has $k$ edge-disjoint spanning branchings rooted in $R_{1}, \cdots, R_{k}$.

Proof. Let $\mathcal{B}_{1}, \cdots, \mathcal{B}_{k}$ be t-edge-disjoint temporal-spanning branchings rooted in $R_{1}, \cdots, R_{k}$, respectively. Partition the edges in $E\left(H_{k}\right) \backslash E_{T}$ into $E_{1}, \ldots, E_{k}$ such that $E_{i}$ has a unique edge from $(u, t)$ to $(u, t+1)$ whenever $\{(u, t),(u, t+1)\} \subseteq V_{T}$. For $i \in\{1, \ldots, k\}$, let $B_{i}^{\prime}$ be a spanning subgraph of $H_{k}$ containing the temporal edges of $\mathcal{B}_{i}$ together with the edges in $E_{i}$. Let $S=s_{1}, \ldots, s_{k}$ be a $s_{1}, s_{k}$-temporal walk in $B_{i}$. We can obtain a $s_{1}, s_{k}$-walk in $H$ from $S$ by adding to $S$ the edge from $(u, t)$ to $(u, t+1)$ in $E_{i}$ between $s_{i}$ and $s_{i+1}$ whenever $s_{i}=(u, t)$ and $s_{i+1}=(u, t+1)$ for any choices of $i \in\{1, \ldots, k-1\}, u \in V(G)$ and $t \in \mathcal{T}(\mathcal{G})$. This implies that $\left\{B_{i}^{\prime}: i \in\{1, \ldots, k\}\right\}$ is a collection of edge disjoint subdigraphs of $H_{k}$ such that every $B_{i}^{\prime}$ contains a branching with root $R_{i}$.

The converse can be proved by deleting the edges in $E\left(H_{k}\right) \backslash E_{T}$ from the disjoint branchings of $H_{k}$ to find the disjoint temporal branchings of $\mathcal{G}$.

\subsubsection{Linearity of $k$-branchings on DAGs}

In this section, given a DAG $G$, a positive integer $k$, and a vertex $r \in V(G)$, we prove that deciding whether $G$ has $k$ edge-disjoint spanning branchings rooted in $r$ can be done in time $O(m+n)$; the branchings can also be produced in the same time if they exist. This is done simply by using a topological order of the vertices of $G$. Observe that, if we have multiple roots, the same argument as the one used in Lemma 3 can be applied.

Lemma 7. Let $G$ be a DAG on $n$ vertices and $m$ edges, $r \in V(G)$ be a source in $G$, and $k$ be a positive integer. Then, one can construct $k$ edge-disjoint spanning branchings of $G$ rooted in $r$ in time $O(m+n)$ if they exist, or provide a proof that they do not exist.

Proof. We prove that $G$ has $k$ edge-disjoint spanning branchings rooted in $r$ if and only if $d^{-}(v) \geqslant k$ for every $v \in V(G) \backslash\{r\}$, giving a simple algorithm that produces the branchings 
if they exist. The necessity comes from the fact that a vertex $v \in V(G) \backslash\{r\}$ has exactly one in-neighbor in each of the $k$ edge-disjoint spanning branchings. To prove sufficiency, let $\left(r, v_{1} \cdots, v_{n}\right)$ be a topological order of the vertices of $G$ obtained in $O(m+n)$ time using a depth-first-search. For each $i \in\{1, \cdots, n\}$, denote by $G_{i}$ the digraph $G\left[\left\{r, v_{1}, \cdots, v_{i}\right\}\right]$, and let $G_{0}=G[\{r\}]$. We build the $k$ edge-disjoint spanning branchings by growing spanning branchings of $G_{i}$, for each $i$. Suppose at step $i$ we have $k$ edge-disjoint spanning branching of $G_{i-1}$. Because we have a topological order of $V(G)$, we know that all the in-neighbors of $v_{i}$ appear before $v_{i}$, i.e., that $d_{G_{i}}^{-}\left(v_{i}\right)=d_{G}^{-}\left(v_{i}\right) \geqslant k$. Therefore, we can build $k$-edge disjoint spanning branchings of $G_{i}$ simply by choosing a distinct edge incident to $v_{i}$ to put in each of the spanning branchings of $G_{i-1}$.

Observe that, if either each snapshot of $\mathcal{G}$ is a DAG or if each edge of $\mathcal{G}$ has a delay, then $H_{k}$ is a DAG. Therefore, we can use Lemmas 6 and 7 to prove Item 1 of Theorem 1.

\subsection{Edge-disjoint Temporal-Spanning Branchings}

In this section, we prove Item 2 of Theorem 1. For this, we first prove that the problem is NP-complete, and then that it is polynomial when each vertex is active for a consecutive set of time stamps. This includes the popular case where vertices are assumed to be permanent, as well as the case where $T(\mathcal{G})=2$.

Theorems 8 and 9 below detail our NP-completeness results. In the next proof, we make a reduction from the $k$-EDGE-DisJOINT PATHS problem (denoted henceforth by $k$-EDP for short), where we are given a digraph $G$ and a set $I$ of $k$ pairs of vertices $\left\{\left(s_{1}, t_{1}\right), \ldots,\left(s_{k}, t_{k}\right)\right\}$ (called the requests) of $V(G)$ and the goal is to find a collection of pairwise edge-disjoint paths $\left\{P_{1}, \ldots, P_{k}\right\}$ such that $P_{i}$ is a path from $s_{i}$ to $t_{i}$ in $G$, for each $i \in\{1, \ldots, k\}$. The $k$-EDP problem is NP-complete for fixed $k \geqslant 2$ [12] and W[1]-hard when parameterized by $k$ even in DAGs [27].

Theorem 8. Let $k \geqslant 2$ be a fixed integer, $\mathcal{G}=(G, \gamma, \lambda)$ be a temporal digraph, and $R_{1}, \ldots, R_{k} \subseteq V_{T}$. Deciding whether $\mathcal{G}$ has $k$ edge-disjoint temporal-spanning branchings rooted in $R_{1}, \cdots, R_{k}$ is NP-complete even if $\mathcal{G}$ has lifetime 3.

Proof. Let $(G, I)$ be an instance of 2 -EDP with $I=\left\{\left(s_{1}, t_{1}\right),\left(s_{2}, t_{2}\right)\right\}$, and define $W=$ $\left\{s_{1}, t_{1}, s_{2}, t_{2}\right\}$. We may assume that $s_{1}, s_{2}$ are sources, $t_{1}, t_{2}$ are sinks, and all vertices in $W$ are distinct. We construct the temporal digraph $\mathcal{G}=(G, \gamma, \lambda)$ with subsets $R_{1}, R_{2}$ such that $\mathcal{G}$ has 2 edge-disjoint temporal-spanning branchings rooted in $R_{1}, R_{2}$ if and only if $(G, I)$ is a "yes" instance of 2-EDP. The NP-completeness for higher values of $k$ follows from Proposition 5.

We let the first snapshot of $\mathcal{G}$ initially consist of $G-\left\{s_{2}, t_{2}\right\}$, and the third snapshot initially consist of $G-\left\{s_{1}, t_{1}\right\}$. Then, we add a new vertex $x$ to snapshot 1 , and add the edges: $\left\{x v \mid v \in V(G) \backslash\left\{s_{2}, t_{2}\right\}\right\} \cup\left\{t_{1} v \mid v \in(V(G) \cup\{x\}) \backslash\left\{s_{1}, s_{2}, t_{2}\right\}\right\}$. Similarly, we add a new vertex $y$ to snapshot 3, and add the edges: $\left\{y v \mid v \in V(G) \backslash\left\{s_{1}, t_{1}\right\}\right\} \cup\left\{t_{2} v \mid\right.$ $\left.v \in(V(G) \cup\{y\}) \backslash\left\{s_{2}, s_{1}, t_{1}\right\}\right\}$. Observe Figure 5 .

Define $R_{1}=\left\{\left(s_{1}, 1\right),(y, 3)\right\}$ and $R_{2}=\left\{\left(s_{2}, 3\right),(x, 1)\right\}$. Now, we prove that $(G, I)$ is a "yes" instance of 2 -EDP if and only if $\mathcal{G}$ contains two edge-disjoint temporal-spanning 

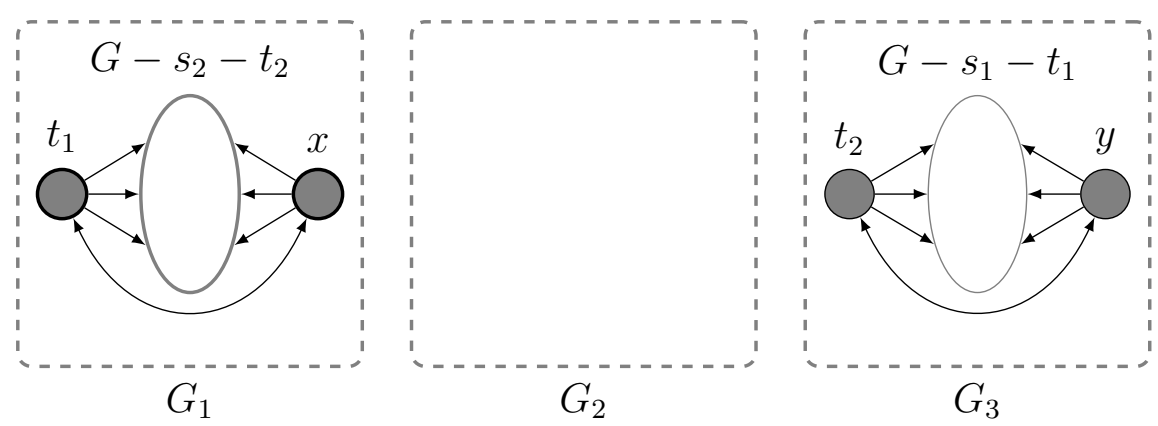

Figure 5: Temporal digraph constructed from an instance $(G, I)$ of 2-EDP, where $I=$ $\left\{\left(s_{1}, t_{1}\right),\left(s_{2}, t_{2}\right)\right\}$ and $W=\left\{s_{1}, t_{1}, s_{2}, t_{2}\right\}$. Edges arriving in $t_{1}$ and $t_{2}$ originally from $G$ are omitted.

branchings rooted in $R_{1}$ and $R_{2}$, respectively. Notice that snapshot 2 of $\mathcal{G}$ is empty, thus each path in $G$ can be represented by either a temporal path on snapshot 1 or a temporal path on snapshot 2.

First, let $P_{1}$ and $P_{2}$ be two edge-disjoint paths from $s_{1}$ to $t_{1}$ and from $s_{2}$ to $t_{2}$ in $G$, respectively. Let $T_{1}$ be initially the copy of $P_{1}$ in snapshot 1 , and $T_{2}$ be initially the copy of $P_{2}$ in snapshot 3 . Note that the vertices not spanned by $T_{1}$ are all the copies of $v \notin V\left(P_{1}\right)$ in snapshot 1 , together with all the vertices in snapshot 3. To span snapshot 3 , add to $T_{1}$ all edges between $(y, 3)$ and $(v, 3)$, for every $v \in V(G) \backslash\left\{s_{1}, t_{1}\right\}$. To span the remainder of snapshot 1 , add all edges between $\left(t_{1}, 1\right)$ and $(v, 1)$, for every $v \in V(G) \backslash\left(V\left(P_{1}\right) \cup\left\{s_{2}, t_{2}\right\}\right)$, and the edge from $\left(t_{1}, 1\right)$ to $(x, 1)$. A similar argument can be applied to span every temporal vertex also with $T_{2}$. Because $P_{1}$ and $P_{2}$ are edgedisjoint, we get that $T_{1}$ and $T_{2}$ could only intersect in the added edges, which does not occur because all edges added to $T_{1}$ are incident to $t_{1}$ and $y$, all edges added to $T_{2}$ are incident to $t_{2}$ and $x$, and there is no intersection between these.

Now, let $T_{1}$ and $T_{2}$ be edge-disjoint temporal-spanning branchings in $\mathcal{G}$ with roots $R_{1}, R_{2}$. Denote snapshot 1 by $G_{1}$. Since $t_{1}$ appears only in $G_{1}$, and the only root of $R_{1}$ in $G_{1}$ is $\left(s_{1}, 1\right)$, we get that in $T_{1}$ there exists a path of $G_{1}$ going from $\left(s_{1}, 1\right)$ to $\left(t_{1}, 1\right)$. Because the only incoming edge to $(x, 1)$ is $\left(t_{1}, 1\right)(x, 1)$, we get that $(x, 1)$ cannot be an internal vertex in this path, and hence it corresponds to a path in $G, P_{1}$. Applying a similar argument, we get a path $P_{2}$ from $s_{2}$ to $t_{2}$ in $G$ taken from $T_{2}$, and since $T_{1}$ and $T_{2}$ are edge-disjoint, so are $P_{1}$ and $P_{2}$.

The next result concludes the NP-completeness results of Item 2 of Theorem 1 . We first prove NP-completeness when no temporal edges have delay, and later we show that we can add delay in an arbitrary number of them with no impact in the reduction. This proves that having delay on the edges does not make the problem easier, contrarily to what happens in the case of $t$-edge-disjoint temporal-spanning.

Theorem 9. Let $k \geqslant 2$ be a fixed integer, $\mathcal{G}=(G, \gamma, \lambda)$ be a temporal digraph, and $R_{1}, \ldots, R_{k} \subseteq V_{T}$. Deciding whether $\mathcal{G}$ has $k$ edge-disjoint temporal-spanning branchings 
rooted in $R_{1}, \cdots, R_{k}$ is NP-complete, even if $G$ is an in-star, each snapshot has constant size, and an arbitrary number of temporal edges have a delay. Furthermore, in this case, there is no algorithm running in time $O^{*}\left(2^{o(T(\mathcal{G}))}\right)$ to solve the problem, unless ETH fails.

Proof. To prove NP-completeness when the underlying graph is an in-star, we make a reduction from NAE-3-SAT, which consists of, given a CNF formula $\phi$ such that each clause has exactly three literals, deciding whether there exists a satisfying truth assignment for $\phi$ such that each clause has at least one true literal and one false literal. This problem is NP-complete [24], and in fact it is a well known standard procedure to make a reduction from 3-SAT to NAE-3-SAT that produces a formula of size linear on the size of the original 3-SAT formula (see e.g. [22], Section 5.3.1). Because, as we will see, our reduction also produces an instance $\mathcal{G}$ such that $T(\mathcal{G})$ is linear on the size of $\phi$, and each snapshot has constant size, we can conclude that our problem cannot be solved in time $O^{*}\left(2^{o(T(\mathcal{G}))}\right)$, unless ETH fails. For more details about ETH and parameterized complexity, we refer the reader to $[9]$.

Let $\phi$ be a CNF formula on variables $\left\{x_{1}, \ldots, x_{n}\right\}$ and clauses $\left\{c_{1}, \ldots, c_{m}\right\}$. The first $n$ odd snapshots are related to variables, and the latter $m$ odd snapshots are related to clauses; we therefore get $2(n+m)-1$ snapshots in total, with the even snapshots being empty (no vertices or edges are active). Let $G$ have vertex set $\{T\} \cup\left\{x_{i}, \bar{x}_{i} \mid i \in\right.$ $\{1, \ldots, n\}\}$, and edge set $\left\{x_{i} T, \bar{x}_{i} T \mid i \in\{1, \ldots, n\}\right\}$. As previously said, the constructed temporal graph $\mathcal{G}$ has lifetime $2(n+m)-1$, and is such that all even snapshots are empty. As for the odd snapshots, for each $i \in\{1, \ldots, n\}$, the $(2 i-1)$-th snapshot is related to variable $x_{i}$, and consists of the following vertices and edges (see Figure 6):

$$
\begin{gathered}
V_{i}=\left\{x_{i}, \bar{x}_{i}, T\right\}, \text { and } \\
E_{i}=\left\{x_{i} T, \bar{x}_{i} T\right\} .
\end{gathered}
$$

Also, add to the sets of roots, $R_{1}, R_{2}$, the temporal vertices $\left(x_{i}, 2 i-1\right)$ and $\left(\bar{x}_{i}, 2 i-1\right)$. As for the remaining odd snapshots, for each $i \in\{1, \ldots, m\}$, let the $(2(n+i)-1)$-th snapshot be related to clause $c_{i}=\left(\ell_{i_{1}} \vee \ell_{i_{2}} \vee \ell_{i_{3}}\right)$ and let it consist of the following vertices and edges (see Figure 7):

$$
\begin{gathered}
C_{i}=\left\{\ell_{i_{1}}, \ell_{i_{2}}, \ell_{i_{3}}, T\right\}, \text { and } \\
E_{i}^{\prime}=\left\{\ell_{i_{1}} T, \ell_{i_{2}} T, \ell_{i_{3}} T\right\} .
\end{gathered}
$$

As before, add the temporal vertices $\left(\ell_{i_{1}}, 2(n+i)-1\right),\left(\ell_{i_{2}}, 2(n+i)-1\right)$ and $\left(\ell_{i_{3}}, 2(n+\right.$ $i)-1)$ to the set of roots, $R_{1}, R_{2}$. Observe then that all appearances of the vertices of $V(G)$ are roots, except for the appearances of vertex $T$. This means that we need only to span the appearances of $T$, which is active in every odd snapshot.

We note that $G$ is an in-star, and each snapshot has constant size. Also, the lower bound on the complexity of an algorithm to solve the problem follows because the size of $\mathcal{G}$ is $O(T(\mathcal{G}))=O(m+n)$. It remains to prove that $\phi$ is a "yes" instance of NAE-3-SAT if and only if $\mathcal{G}$ has two edge-disjoint temporal-spanning branchings $\mathcal{B}_{1}$ and $\mathcal{B}_{2}$ rooted in $R_{1}=R_{2}$. The theorem holds for bigger values of $k$ by Proposition 5 . 
Suppose that $\phi$ is a "yes" instance of NAE-3-SAT. For each true variable $x_{i}$, put all occurences of the edge $x_{i} T$ in $\mathcal{B}_{1}$, and all occurences of the edge $\bar{x}_{i} T$ in $\mathcal{B}_{2}$. For each false variable, do as before, switching between $\mathcal{B}_{1}$ and $\mathcal{B}_{2}$. Figure 6 represents $\mathcal{B}_{1}$ with solid edges and $\mathcal{B}_{2}$ with dotted edges, when $x_{i}$ is true. Now, consider a clause $c_{i}=\left(\ell_{i_{1}} \vee \ell_{i_{2}} \vee \ell_{i_{3}}\right)$. Because this is a NAE assignment, we know that there is at least one true literal, say $\ell_{i_{1}}$, and one false literal, say $\ell_{i_{2}}$. This means that temporal edges related to $\ell_{i_{1}} T$ can only be in $\mathcal{B}_{1}$, and edges related to $\ell_{i_{2}} T$ can only be in $\mathcal{B}_{2}$. Therefore, for each $i \in\{1, \ldots, m\}$, we can span temporal vertex $(T, 2(n+i)-1)$ in both $\mathcal{B}_{1}$ and $\mathcal{B}_{2}$ by using these edges (recall that $\left\{\left(\ell_{i_{1}}, 2(n+i)-1\right),\left(\ell_{i_{2}}, 2(n+i)-1\right)\right\} \subseteq R_{1}=R_{2}$, where $\ell_{i_{1}}, \ell_{i_{2}}, \ell_{i_{3}}$ are the literals in clause $\left.c_{i}\right)$. Figure 7 represents the branching related to the assignment $(F, F, T)$ for $\left(x_{i_{1}}, x_{i_{2}}, x_{i_{3}}\right)$ in the clause $\left(\bar{x}_{i_{1}} \vee x_{i_{2}} \vee x_{i_{3}}\right)$.

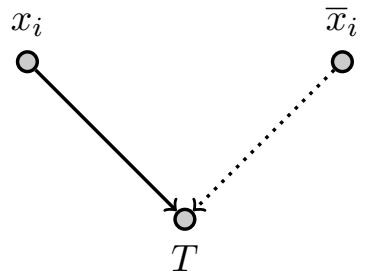

Figure 6: Branchings in a variable gadget. Figure 7: Branchings in a clause gadget.

Now, let $\mathcal{B}_{1}, \mathcal{B}_{2}$ be two edge-disjoint temporal-spanning branchings rooted in $R_{1}=R_{2}$, and denote by $B_{i}$ the set of temporal edges of $\mathcal{B}_{i}$. For each variable $x_{i}$, let $x_{i}$ be true if and only if $\left(x_{i} T, 2 i-1\right) \in B_{1}$. Now, consider a clause $c_{i}=\left(\ell_{i_{1}} \vee \ell_{i_{2}} \vee \ell_{i_{3}}\right)$, and denote $2(n+i)-1$ by $j$. Because $(T, j)$ must be spanned by $B_{1}$ and $B_{2}$, then at least one of the edges incoming to $(T, j)$ must be in $B_{1}$, say $\left(\ell_{i_{1}} T, j\right)$, and at least one must be in $B_{2}$, say $\left(\ell_{i_{2}} T, j\right)$. But this means that $\left(\ell_{i_{1}} T, 2 i_{1}-1\right) \in B_{1}$ (hence $\ell_{i_{1}}$ is a true literal), and that $\left(\ell_{i_{2}} T, 2 i_{2}-1\right) \in B_{2}$ (hence $\ell_{i_{2}}$ is a false literal), as we wanted to show.

Finally, one can see that if we stretch the temporal digraph, we can add delay in any of the edges in the gadgets. It suffices to make a gadget consist of two snapshots, the first one containing the vertices related to the variables, and the second one containing vertex $T$, and making the edges between the former vertices and the latter have a delay of one.

The following theorem gives us a situation where the problem becomes easy. Note that this case includes the permanent temporal digraphs, which are used in $[1,6,16,20,23]$.

Theorem 10. Let $\mathcal{G}=(G, \gamma, \lambda)$ be a temporal digraph, and let $R_{1}, \cdots, R_{k} \subseteq V_{T}$. If for every $v \in V(G), \gamma(v)$ is exactly one interval of consecutive integers, then finding $k$ edgedisjoint temporal-spanning branchings rooted in $R_{1}, \cdots, R_{k}$ can be done in polynomial time.

Proof. To prove this theorem, we build digraphs $F_{1}, \ldots, F_{T(\mathcal{G})}$ and sets $R_{1}^{j}, \cdots, R_{k}^{j}$ for each $j \in \mathcal{T}(\mathcal{G})$. Then, we prove that $\mathcal{G}$ has the desired branchings if and only if $F_{j}$ has $k$ 
edge-disjoint branchings rooted in $R_{1}^{j}, \ldots, R_{k}^{j}$ for each $j \in \mathcal{T}(\mathcal{G})$. This can be checked in polynomial time, applying Edmonds' Theorem [10].

For each $j \in \mathcal{T}(\mathcal{G})$, we build $F_{j}$ in the following way. Let $V\left(F_{j}\right)$ be initially equal to the set of vertices that are active in time stamp $j$, i.e., $F_{j}$ initially contains only $\{u \in V(G) \mid$ $\left.(u, j) \in V_{T}\right\}$. Then, add to $F_{j}$ all vertices which are the tail of some edge arriving in time stamp $j$, i.e., add the set $\left\{u \in V(G) \mid(u v, t, j) \in E_{T}\right.$ for some $\left.v, t\right\}$. Finally let $F_{j}$ contain all edges arriving in time $j$, i.e., $E\left(F_{j}\right)$ is the set $\left\{e \in E(G) \mid(e, t, j) \in E_{T}\right.$ for some $\left.t\right\}$. For each $j \in \mathcal{T}(\mathcal{G})$ and each $i \in\{1, \cdots, k\}$, let $R_{i}^{j}$ be the set of roots at time stamp $j$ from the set $R_{i}$ together with any vertex of $V\left(F_{j}\right)$ active in a previous time stamp, i.e., $R_{i}^{j}=\left\{u \in V\left(F_{j}\right) \mid(u, j) \in R_{i}\right.$ or $\left.\{1, \ldots, j-1\} \cap \gamma(u) \neq \emptyset\right\}$.

We now prove that $\mathcal{G}$ has the desired branchings if and only if $F_{j}$ has $k$ edge-disjoint branchings rooted in $R_{1}^{j}, \ldots, R_{k}^{j}$ for each $j \in \mathcal{T}(\mathcal{G})$. First, let $\mathcal{B}_{1}, \ldots, \mathcal{B}_{k}$ be edge-disjoint temporal-spanning branchings rooted in $R_{1}, \cdots, R_{k}$. Consider $j \in \mathcal{T}(\mathcal{G})$, and for each $i \in\{1, \cdots, k\}$, let $B_{i}^{j}$ be the spanning subgraph of $F_{j}$ with edge set being the edges of $\mathcal{B}_{i}$ that has a copy ending at time stamp $j$, i.e., $E\left(B_{i}^{j}\right)=\left\{e \in E\left(F_{j}\right) \mid(e, t, j) \in\right.$ $E_{T}\left(\mathcal{B}_{i}\right)$ for some $\left.e \in E(G), t \in \mathcal{T}(\mathcal{G})\right\}$. Since $\mathcal{B}_{1}, \ldots, \mathcal{B}_{k}$ are edge-disjoint, we get that $B_{1}^{j}, \ldots, B_{k}^{j}$ are also edge-disjoint. It remains to prove that each $B_{i}^{j}$ is a branching of $F_{j}$ rooted in $R_{i}^{j}$. To do so, we prove that if $u \in V\left(F_{j}\right)$ is a source in $B_{i}^{j}$, then $u \in R_{i}^{j}$. Indeed, this is the case since, if $u$ is not the head of any edge in $B_{i}^{j}$ and $(u, j) \notin R_{i}$, then either $j \in \gamma(u)$ and $(u, j)$ is spanned in $\mathcal{B}_{i}$ just by waiting or $j \notin \gamma(u)$ and $u$ is added to $F_{j}$ through an edge arriving at time $j$. In either case we have $\{1, \ldots, j-1\} \cap \gamma(u) \neq \emptyset$.

Now, for each $j \in \mathcal{T}(\mathcal{G})$, let $B_{1}^{j}, \ldots, B_{k}^{j}$ be $k$ edge-disjoint spanning branchings of $F_{j}$. For each $i \in\{1, \ldots, k\}$, let $B_{i}=\bigcup_{j=1}^{T(\mathcal{G})} B_{i}^{j}$, and $\mathcal{B}_{i}=\left(B_{i}, \gamma, \lambda^{i}\right)$ be a spanning temporal subdigraph of $\mathcal{G}$ where $\lambda^{i}$ is the restriction of $\lambda$ to the domain $E\left(B_{i}\right)$. Observe that $B_{1}, \ldots, B_{k}$ are edge disjoint. Indeed, consider an edge $e \in E(G)$ with head $v$ and let $j \in \mathcal{T}(\mathcal{G})$ be minimum such that $e \in B_{i}^{j}$ for some $i \in\{1, \cdots, k\}$. Since $B_{i}^{j} \cap B_{i^{\prime}}^{j}=\emptyset$ for every $i^{\prime} \neq i$, and by the choice of $j$, we get that $e \notin B_{i^{\prime}}^{j^{\prime}}$ for every $j^{\prime} \leqslant j$ and every $i^{\prime} \neq i$. Furthermore, since $j \in \gamma(v)$, we have that $v \in V\left(F_{j^{\prime}}\right)$ implies $v$ is in the root of every subsequent branching, and hence $v$ has no incoming edges in $B_{i^{\prime}}^{j^{\prime}}$ for every $j^{\prime}>j$ and every $i^{\prime} \in\{1, \ldots, k\}$. It thus follows that $\mathcal{B}_{1}, \cdots, \mathcal{B}_{k}$ are edge-disjoint, so it remains to prove that each $\mathcal{B}_{i}$ is a temporal-spanning branching rooted in $R_{i}$. Let $u \in V(G)$ and let $j$ be the smallest value in $\gamma(u)$. Note that we just need to prove that if $(u, j) \notin R_{i}$, then there exists a temporal edge in $\mathcal{B}_{i}$ arriving in $(u, j)$ since the other temporal copies can be spanned simply by waiting in the interval $\gamma(u)$. Thus, suppose $(u, j) \notin R_{i}$. Now, we get that $u \notin R_{i}^{j}$ as $\{1, \ldots, j-1\} \cap \gamma(u)=\emptyset$ by the choice of $j$. Since $B_{i}^{j}$ is a spanning branching of $F_{j}$ rooted at $R_{i}^{j}$, we get that $B_{i}^{j}$ contains an edge into $u$. This implies that there exists a temporal edge in $\mathcal{B}_{i}$ arriving in $(u, j)$.

The only missing cases of Item 2 of Theorem 1 are the ones concerning linearity for permanent temporal digraphs where all edges have a delay, or where each snapshot is a DAG. Observe that the proof of the above theorem tells us that, in order to solve the problem on a permanent temporal digraph $\mathcal{G}$, it suffices to solve $T(\mathcal{G})$ instances of the $k$ edge-disjoint branchings problems. By Lemma 7, we know that each such instance can 
be solved in linear time, and since the size of $\mathcal{G}$ is the sum of the sizes of the snapshots, Item 2 of Theorem 1 follows.

\section{Vertex-spanning Branchings}

In this section, we provide an NP-completeness proof for both Item 3 and Item 4 of Theorem 1. Again, we make a reduction from NAE-3-SAT.

Let $\phi$ be a CNF formula on variables $\left\{x_{1}, \ldots, x_{n}\right\}$ and clauses $\left\{c_{1}, \ldots, c_{m}\right\}$. A variable gadget related to $x_{i}$ is formed by the set of vertices $V_{i}=\left\{x_{i}, F_{i}, T_{i}, a_{i}\right\}$ and the set of edges $E_{i}=\left\{x_{i} T_{i}, x_{i} F_{i}, T_{i} a_{i}, F_{i} a_{i}\right\}$ (see Figure 8).

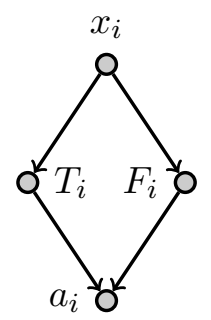

Figure 8: Gadged related to variable $x_{i}$.

Now, consider a clause $c_{i}=\left\{\ell_{i_{1}}, \ell_{i_{2}}, \ell_{i_{3}}\right\}$, and for each $i \in\{1,2,3\}$ let $x_{i_{j}}$ be the variable related to literal $\ell_{i_{j}}$. Add vertex $c_{i}$ to the constructed graph. Then, for each $i \in\{1,2,3\}$, if $x_{i_{j}}$ appears positively in $c_{i}$, then add edge $T_{i_{j}} c_{i}$ to the clause gadget related to $c_{i}$; otherwise, add edge $F_{i_{j}} c_{i}$. Denote by $C_{i}$ the set of vertices in the clause gadget of $c_{i}$, and by $E_{i}^{\prime}$, the set of edges. Now, let $G_{\phi}$ be the digraph formed by the union of all variable and clause gadgets, i.e., $V(G)=\bigcup_{i=1}^{n} V_{i} \cup \bigcup_{i=1}^{m} C_{i}$ and $E(G)=\bigcup_{i=1}^{n} E_{i} \cup \bigcup_{i=1}^{m} E_{i}^{\prime}$. Add to $G_{\phi}$ two new vertices, $g, r$, and add edges $\left\{g x_{i}, r x_{i}\right\}$ for every $i \in\{1, \cdots, n\}$. Finally, let $G^{\prime}$ be the digraph having $A \cup\{g, r\}$ as vertex set, where $A=\left\{T_{i}, F_{i} \mid i \in\{1, \cdots, n\}\right\}$, and having every edge going from $\{g, r\}$ to $A$. Let $\mathcal{G}$ be the temporal digraph with lifetime 2 , having $G_{\phi}$ as first snapshot and $G^{\prime}$ as second snapshot (therefore, the base digraph of $\mathcal{G}$ is given by $\left(V, E\left(G_{\phi}\right) \cup E\left(G^{\prime}\right)\right)$, where $\left.V=V\left(G_{\phi}\right) \supset V\left(G^{\prime}\right)\right)$. See Figure 9 for the temporal digraph related to $\phi=\left(x_{1} \vee \bar{x}_{2} \vee x_{3}\right)$.

Theorem 11. For each $k \geqslant 2$, given a temporal digraph $\mathcal{G}=(G, \gamma, \lambda)$ and subsets $R_{1}, \cdots, R_{k} \subseteq V_{T}$, it is NP-complete to decide whether $\mathcal{G}$ has $k$ (t-edge-disjoint or edgedisjoint) vertex-spanning branchings rooted in $R_{1}, \cdots, R_{k}$, even if $G$ is a DAG, vertices are permanent, and either $T(\mathcal{G})=2$ and all edges are instantaneous, or $T(\mathcal{G})=5$ and all edges have a delay. Furthermore, letting $n=|V(G)|$ and $m=|E(G)|$, no algorithm running in time $O^{*}\left(2^{o(n+m)}\right)$ can exist for the problem, unless ETH fails.

Proof. We first consider the case that no edges have delay, and at the end we see that the digraph can be stretched in order to add delay to all edges. We prove the theorem for $k=2$, and NP-completeness for bigger values of $k$ follows by Proposition 5 . Let $\phi$ be an 


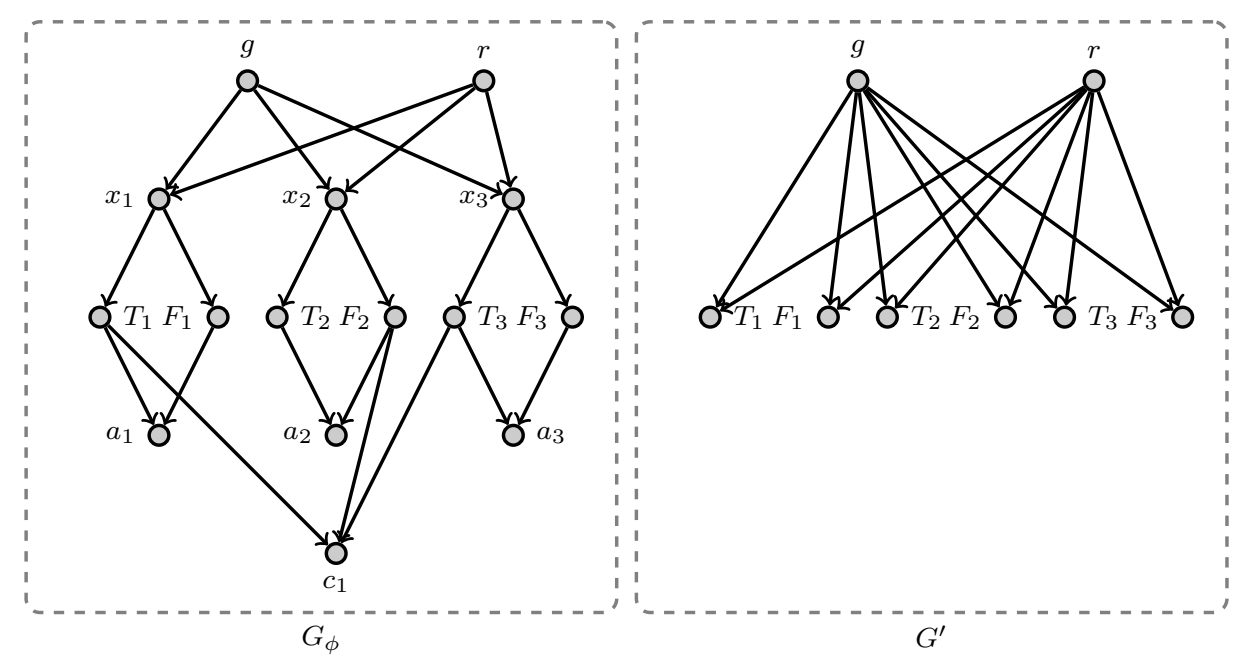

Figure 9: Temporal digraph related to $\phi=\left(x_{1} \vee \bar{x}_{2} \vee x_{3}\right)$.

instance of NAE-3-SAT on variables $\left\{x_{1}, \cdots, x_{n}\right\}$ and clauses $\left\{c_{1}, \cdots, c_{m}\right\}$, and let $\mathcal{G}$ be the temporal digraph constructed as before; denote by $G$ the base digraph. The second part follows easily since the reduction gives rise to a temporal digraph of size $O(n+m)$. In what follows, we prove that $\phi$ is a "yes" instance of NAE-3-SAT if and only if $\mathcal{G}$ has $k$ edge-disjoint vertex-spanning branchings rooted in $\{(g, 1),(r, 1)\}$. Observe that, since there is no edge occurring in both timestamps, the proof also holds for t-edge-disjoint branchings.

First, suppose that $\phi$ is a "yes" instance of NAE-3-SAT. We construct edge-disjoint vertex-spanning branchings $\mathcal{B}_{1}$ and $\mathcal{B}_{2}$ rooted in $\{(g, 1),(r, 1)\}$. For each true variable $x_{i}$, add to $\mathcal{B}_{1}$ the following edges of snapshot $1:\left\{g x_{i}, x_{i} T_{i}, T_{i} a_{i}\right\}$, together with edge $T_{i} c_{j}$ for each clause $c_{j}$ containing $x_{i}$ that is not reached by $\mathcal{B}_{1}$ yet; also add to $\mathcal{B}_{2}$ edges $\left\{r x_{i}, x_{i} F_{i}, F_{i} a_{i}\right\}$, together with edge $F_{i} c_{j}$ for each clause $c_{j}$ containing $\bar{x}_{i}$ that is not reached by $\mathcal{B}_{2}$ yet. Do something similar to the false variables, but switching the branchings. Figure 10 gives the branchings related to the assignment $(T, T, F, F)$ to $\left(x_{1}, x_{2}, x_{3}, x_{4}\right)$, respectively (they are represented by the black and dotted edges). Observe that every $u \in V(G)$ is spanned by both branchings, with the exception of vertices in $B=\left\{T_{i}, F_{i} \mid\right.$ $i \in\{1, \cdots, n\}\}$ since each $u \in B$ is spanned by exactly one between $\mathcal{B}_{1}$ and $\mathcal{B}_{2}$. However, these can easily be spanned in the second snapshot since $\{g, r\}$ is complete to $B$.

Now, let $\mathcal{B}_{1}, \mathcal{B}_{2}$ be two edge-disjoint vertex-spanning branchings. Since each $a_{i}$ can only be reached at the first snapshot, it is reached by exactly two paths from $\{(g, 1),(r, 1)\}$, one of them going through $\left(x_{i}, 1\right)\left(T_{i}, 1\right)$ and the other through $\left(x_{i}, 1\right)\left(F_{i}, 1\right)$. We then put $x_{i}$ as true if and only if $\left(x_{i}, 1\right)\left(T_{i}, 1\right)$ is in branching $\mathcal{B}_{1}$. Now, consider clause $c_{i}=\left(\ell_{i_{1}} \vee \ell_{i_{2}} \vee \ell_{i_{3}}\right)$. Since $c_{i}$ is spanned by $\mathcal{B}_{1}$ and $\mathcal{B}_{2}$, we get that at least one of the edges in $E_{i}^{\prime}$ is in $\mathcal{B}_{1}$, and at least one in $\mathcal{B}_{2}$, which implies that at least one of $\ell_{i_{1}}, \ell_{i_{2}}, \ell_{i_{3}}$ is true, and at least one is false, as desired.

Finally, observe Figure 9 to see that, if we stretch these paths along the snapshots 


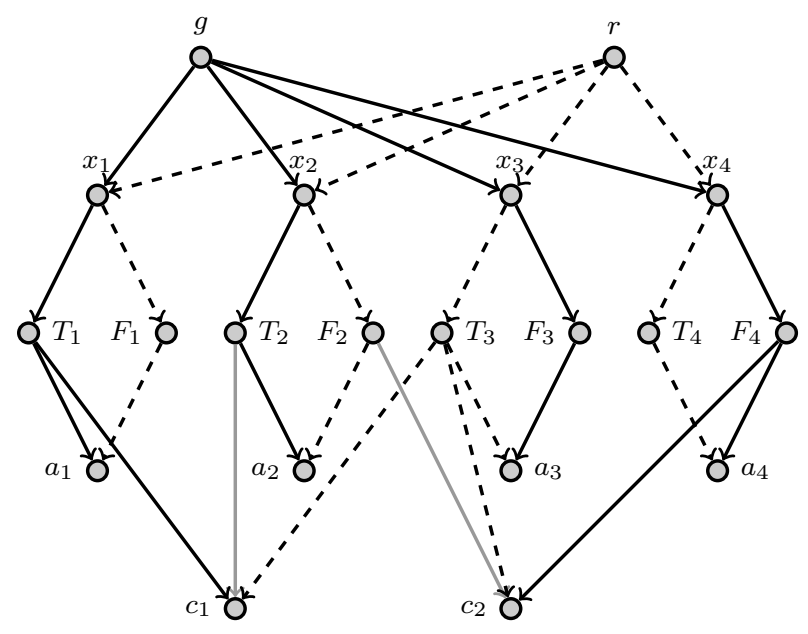

Figure 10: Snapshot 1 related to formula $\phi=\left(x_{1} \vee x_{2} \vee x_{3}\right) \wedge\left(\bar{x}_{2} \vee x_{3} \vee \bar{x}_{4}\right)$, and branchings related to the assignment $(T, T, F, F)$ to $\left(x_{1}, x_{2}, x_{3}, x_{4}\right)$.

we can add delay to every edge. More formally, we consider the temporal digraph with lifetime 5 and put all the edges of type $\left\{g x_{i}, r x_{i}\right\}$ between timestamp 1 and 2 , all edges of type $\left\{x_{i} T_{i}, x_{i} F_{i}\right\}$ between timestamp 2 and 3 , all edges of type $\left\{T_{i} a_{i}, F_{i} a_{i}, T_{j} c_{i}, F_{j} c_{i}\right\}$ between timestamps 3 and 4 , and all edges of type $\left\{g T_{i}, g F_{i}, r T_{i}, r F_{i}\right\}$ between timestamps 4 and 5 .

\section{Discussing natural extensions of Edmonds' Condition}

As said before, Edmonds' Theorem is the characterization behind the polynomial algorithm for finding $k$ edge-disjoint spanning branchings in digraphs [10]. It actually can be stated in many forms. Below, for simplicity, we present the possible statements when only one root is considered.

Theorem 12 (Edmonds [11], as stated by Schrijver [25]). Given a digraph G, a vertex $r \in V(G)$, and a positive integer $k$, the following are equivalent.

1. G has $k$ edge-disjoint spanning branchings rooted in $r$;

2. There are $k$ edge-disjoint paths from $r$ to $v$, for every $v \in V(G) \backslash\{r\}$;

3. There are $k$ edge-disjoint spanning trees in the underlying undirected graph such that, for each $v \neq r$ there are exactly $k$ edges entering $v$ covered by these trees;

4. $d^{-}(U) \geqslant k$ for every $U \subseteq V(G) \backslash\{r\}$.

In order to investigate the validity of Edmonds' Theorem on the temporal context, again we need to consider the various types of edge-disjoint branchings in this context. For simplicity, we consider only branchings rooted at a single vertex. We have seen in 
Section 2 that this has no impact in the complexity of the problem. Also, because the connectivity problems that appear in the literature are mostly concerned about temporal digraphs with permanent vertices, and also usually consider only t-edge-disjoint paths between vertices of $G$, in the following we discuss only the t-edge-disjoint vertex-spanning case on permanent temporal digraphs.

Let $\mathcal{G}=(G, \gamma, \lambda)$ be a temporal digraph, $(r, 1)$ be a temporal vertex of $\mathcal{G}$, and $k$ be a positive integer, $k \geqslant 2$. Below, we discuss, for Items $2-3$ of Theorem 12 , the possibility of a temporal formulation that would be equivalent to the t-edge-disjoint vertex-spanning version of Item 1 . We state this version of Item 1 for facility of reference.

$\left.{ }^{*}\right)(G, \gamma, \lambda)$ has $k$ t-edge-disjoint vertex-spanning branchings rooted in $(r, 1)$.

In [2], the author proved that, given a pair of vertices $u, v \in V(G)$, deciding whether there exist $k$ t-edge-disjoint temporal walks between $u$ and $v$ can be done in polynomial time. Therefore, by Theorem 1, we get that $\left(^{*}\right)$ cannot be equivalent to a temporal version of Item 2 of Theorem 12, unless $\mathrm{P}=\mathrm{NP}$. We mention that in [2], the author also proved that, given vertices $u, v$, deciding whether there are 2 edge-disjoint temporal walks between $u$ and $v$ is NP-complete. This gives us that the edge-disjoint vertex-spanning variation could still hold on permanent temporal digraphs.

As for Item 3, the natural adaptation of the concept of vertex-spanning trees would be that of a temporal subgraph of the underlying temporal graph (the temporal graph obtained simply by ignoring directions of $G$ ) such that, for each $v \in V(G) \backslash\{r\}$, there exists a temporal walk between $(r, 1)$ and $(v, i)$ for some $i \in \mathcal{T}(\mathcal{G})$. Also, note that the number of "edges entering $v$ " should consider temporal edges, as these are the edges being used in the vertex-spanning trees. However, as we have seen in Figure 3 in Section 2, we may need to visit the same vertex of $G$ more than once in a same vertex-spanning branching, implying that the number of temporal edges entering $v$ can be larger than $k$. Therefore, again a temporal version of Item 3 cannot be equivalent to the fact that $\mathcal{G}$ has $k$ t-edge-disjoint vertex-spanning branchings rooted in $(r, 1)$. We mention that this kind of situation also occurs in the other variations of the problem. Therefore, if we consider general temporal digraphs, this version of Edmonds' Theorem does not seem to hold in all of the cases. As for permanent temporal digraphs, this could be a possible formulation since a temporal walk in these digraphs can always be truncated in order to yield temporal paths.

Now, observe that Item 4 (that we call Edmonds' Condition) does not necessarily imply a polynomial algorithm. However, Lovász's proof [18] provides an algorithm that runs in time $O\left(k^{2} m^{2}\right)$, where $m=|E(G)|$. This means that a temporal version of Item 4 could still hold, even if, because of our NP-completeness results, such a condition would not be verifiable in polynomial time unless $P=N P$. Nevertheless, we believe that a characterizing condition that depends only on the (temporal) indegree of subsets of (temporal) vertices is indeed not possible, as we discuss next.

There are two main ingredients in Edmonds' Condition: which sets are of interest, and what should be the indegree of a set. A first attempt could be to consider every subset $X$ of vertices of $G$. Clearly the indegree considered cannot be the indegree of $X$ 
in $G$ since it could happen, for instance, that Edmonds' Condition holds on $G$, but that $(r, 1)$ is isolated in the first snapshot. So alternatively, we could define the indegree of $X$ as the number of temporal edges arriving in any temporal vertex $(x, i)$ such that $x \in X$. But this is not sufficient, since it could happen again that $(r, 1)$ is isolated in the first snapshot, while $X$ receives enough edges to satisfy the condition, and no more edges later on.

So, let us consider subsets of temporal vertices instead. In this case, the indegree must take into account only temporal edges arriving at $X$, since it would make no sense to count on edges that are not active at the same time as $X$. But in this case, the related condition is not necessary as it could happen, for instance, that $V(G)$ can be spanned on the first snapshot, therefore no indegree of a subset $X \subseteq V_{T}$ contained in the second snapshot is needed. This however gives us a clue to yet another possibility, which is to consider temporal edges entering $X$ that occur earlier than $X$. This is a good guess since it is possible to wait on the vertices as they are permanent. For this, we say that a subset $X \subseteq V_{T}$ is a prefix set if whenever $(u, i) \in X$, we get that $\{(u, j) \mid j \leqslant i\} \subseteq X$. The temporal indegree of $X$ in $\mathcal{G}$ is then defined as the number of temporal edges arriving at $X$; more formally, $d_{\mathcal{G}}^{-}(X)=\mid\{(u v, i, j) \mid(u, i) \notin X$ and $(v, j) \in X\} \mid$. A last detail is that we also require that a prefix set contains at least one temporal vertex of the last snapshot. This is because otherwise, it could happen that $(r, 1)$ is isolated throughout the lifetime of $\mathcal{G}$, except that Edmonds' Condition holds on the last snapshot, i.e., the condition would be false for every prefix subset $X$ not intersecting the last snapshot, but the temporal digraph still contains the desired branchings. One can verify that the following condition is necessary, however we will see that it is not sufficient.

$$
d_{\mathcal{G}}^{-}(X) \geqslant|\{i \mid(r, 1) \notin X=\emptyset\}| \text {, for every prefix set } X \subseteq V_{T} .
$$

Consider the example in Figure 11 (we omit the copies of vertices that have indegree 0 in the second snapshot). One can verify that every prefix set $X$ not containing $(r, 1)$ has at least two incoming temporal edges, i.e., Equation 1 holds. Nevertheless, it is not possible to obtain two t-edge-disjoint vertex-spanning branchings. In fact, this example was constructed by a slight modification of the gadget presented in Section 4 applied to the formula $\left(x_{1} \vee x_{2}\right) \vee\left(\bar{x}_{1} \vee x_{2}\right)$, which does not have a NAE satisfying assignment. One can verify that either $\left(c_{1}, 1\right)$ or $\left(c_{2}, 1\right)$ is not spanned by one of the branchings. Up to our knowledge, there are no conditions that ensure the satisfiability of a formula that depend only on the degree of the variables in the clauses, especially without considering global structures (e.g., a subset of variables that covers the clauses). This is why we believe that there is no version of Edmonds' Condition that work on temporal digraphs. We mention that our reductions are also from NAE-3-SAT in the other cases, which means that this version of Edmonds' Theorem also does not seem to hold in the other cases.

\section{Conclusions and open problems}

In this paper we have investigated temporal versions of the classical Edmonds' Theorem about the problem of finding $k$ edge-disjoint spanning branchings of a digraph $G$ rooted in 

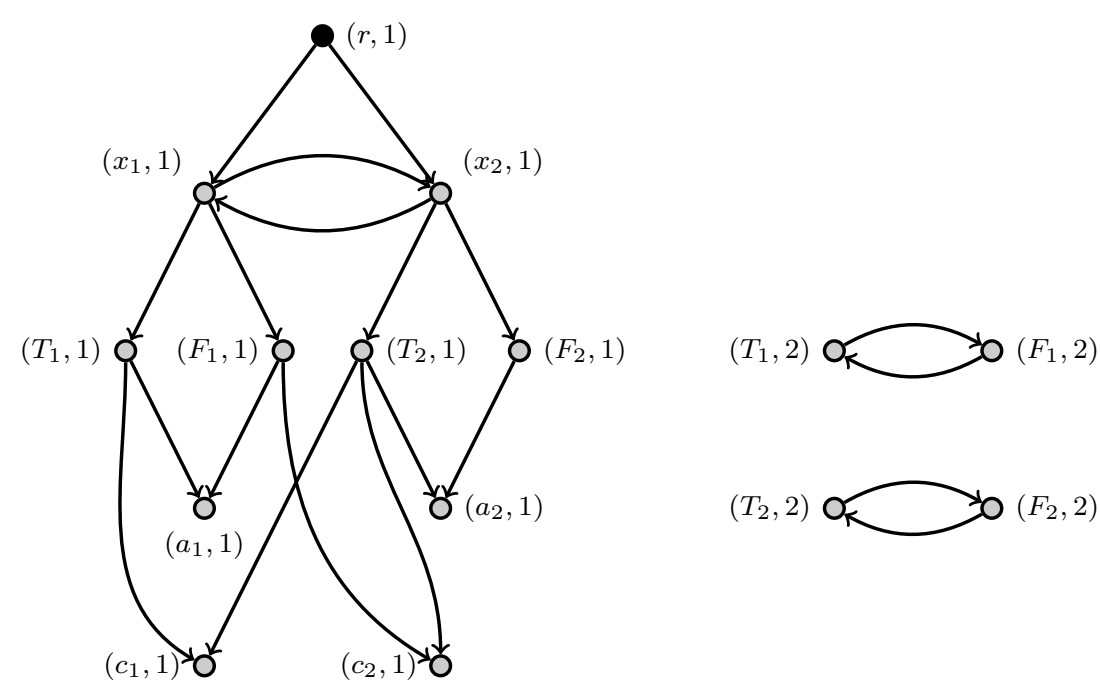

Figure 11: Temporal digraph satisfying Equation 1 but not having disjoint branchings rooted in $(r, 1)$.

given $R_{1}, \cdots, R_{k}$. We have introduced different definitions of spanning branchings and of edge-disjointness in temporal digraphs. We have proved that, unlike the static case, only one of the these can be computed in polynomial time, namely the temporal-edge-disjoint temporal-spanning branchings problem, while the other versions are NP-complete under very strict constraints. Also, if the temporal vertices appear for a continuous window of time, then finding temporal-spanning branchings is polynomial-time solvable, while finding vertex-spanning branchings is NP-complete. This includes temporal digraphs of lifetime two, as well as the more popular model where the vertices are permanent.

Given a temporal digraph $\mathcal{G}=(G, \gamma, \lambda)$, in the particular case of edge-disjoint temporal-spanning branchings, we give separate NP-complete results, one for fixed lifetime and another one for when $G$ is an in-star. We then pose the following question. Given a temporal digraph $\mathcal{G}$ and subsets $R_{1}, \cdots, R_{k}, k \geqslant 2$, does there exist an FPT algorithm, parameterized by the lifetime of $\mathcal{G}$ and the treewidth of $G$, that decides whether $\mathcal{G}$ has $k$ edge-disjoint temporal-spanning branchings rooted in $R_{1}, \cdots, R_{k}$ ?

Observe also that almost all of our NP-completeness results hold when $G$ is a DAG, but only if the lifetime of $\mathcal{G}$ is $O(|V(G)|+|E(G)|)$. Therefore, we also ask whether these problems become polynomial-time solvable when $G$ is a DAG if the lifetime of $\mathcal{G}$ is either constant or a logarithmic function on the size of $G$. And as we have provided computational lower bounds under ETH in Theorem 9 and in Theorem 11, we also ask whether there exist algorithms matching these lower bounds.

Concerning Edmonds' Theorem on digraphs, we showed that two of the possible statements of the theorem do not have an equivalent version on the temporal context. Besides, we discussed about possible temporal versions of a characterization that depends on the (temporal) indegrees of subsets of (temporal) vertices, giving reasons why we do not be- 
lieve that such a characterization exists. We ask whether there exists a characterization that depends on more than the indegrees of temporal subsets.

\section{References}

[1] Eleni Akrida, Jurek Czyzowicz, Leszek Gasieniec, Lukasz Kuszner, and Paul Spirakis. Temporal flows in temporal networks. Journal of Computer and System Sciences, 103:46-60, 2019.

[2] Kenneth Berman. Vulnerability of scheduled networks and a generalization of Menger's Theorem. Networks: An International Journal, 28(3):125-134, 1996.

[3] Sandeep Bhadra and Afonso Ferreira. Complexity of connected components in evolving graphs and the computation of multicast trees in dynamic networks. In Proc. of the 2nd International Conference on Ad-Hoc Networks and Wireless (ADHOCNOW), volume 2865 of LNCS, pages 259-270, 2003.

[4] Adrian Bondy and Uppaluri Murty. Graph Theory. Graduate Texts in Mathematics. Springer London, 2011.

[5] Pierre Borgnat, Eric Fleury, Jean-Loup Guillaume, Clémence Magnien, Céline Robardet, and Antoine Scherrer. Evolving networks. In Mining massive data sets for security, volume 19, pages 198-203. 2008.

[6] Binh-Minh Bui-Xuan, Afonso Ferreira, and Aubin Jarry. Computing shortest, fastest, and foremost journeys in dynamic networks. International Journal of Foundations of Computer Science, 14(2):267-285, 2003.

[7] Victor A. Campos, Raul Lopes, Andrea Marino, and Ana Silva. Edge-disjoint branchings in temporal graphs. In Proc. of the 31st International Workshop on Combinatorial Algorithms (IWOCA), volume 12126 of LNCS, pages 112-125, 2020.

[8] Arnaud Casteigts, Paola Flocchini, Walter Quattrociocchi, and Nicola Santoro. Time-varying graphs and dynamic networks. International Journal of Parallel, Emergent and Distributed Systems, 27(5):387-408, 2012.

[9] Marek Cygan, Fedor V Fomin, Lukasz Kowalik, Daniel Lokshtanov, Dániel Marx, Marcin Pilipczuk, Michał Pilipczuk, and Saket Saurabh. Parameterized algorithms, volume 4. Springer, 2015.

[10] Jack Edmonds. Edge-disjoint branchings. Combinatorial algorithms, 1973.

[11] Jack Edmonds. Some well solved problems in combinatorial optimization. In Combinatorial programming: methods and applications, pages 285-311. 1975.

[12] Steven Fortune, John Hopcroft, and James Wyllie. The directed subgraph homeomorphism problem. Theoretical Computer Science, 10(2):111-121, 1980.

[13] András Frank. Covering branchings. Acta Scientiarium Mathematicarum (Szeged), 41:77-81, 1979.

[14] Delbert Ray Fulkerson and Gary C. Harding. On edge-disjoint branchings. Networks, 6(2):97-104, 1976. 
[15] Silu Huang, Ada Wai-Chee Fu, and Ruifeng Liu. Minimum spanning trees in temporal graphs. In Proc. of the 2015 ACM SIGMOD International Conference on Management of Data (SIGMOD/PODS), pages 419-430, 2015.

[16] David Kempe, Jon Kleinberg, and Amit Kumar. Connectivity and inference problems for temporal networks. Journal of Computer and System Sciences, 64(4):820-842, 2002.

[17] Matthieu Latapy, Tiphaine Viard, and Clémence Magnien. Stream graphs and link streams for the modeling of interactions over time. Social Network Analysis and Mining, 8(1):1-29, 2018.

[18] László Lovász. On two minimax theorems in graph. Journal of Combinatorial Theory, Series B, 21(2):96-103, 1976.

[19] Colin McDiarmid. Integral decomposition in polyhedra. Mathematical Programming, 25(2):183-198, 1983.

[20] George B Mertzios, Othon Michail, Ioannis Chatzigiannakis, and Paul G Spirakis. Temporal network optimization subject to connectivity constraints. Algorithmica, 81(4):1416-1449, 2019.

[21] Othon Michail. An introduction to temporal graphs: an algorithmic perspective. Internet Mathematics, 12(4):239-280, 2016.

[22] Cristopher Moore and Stephan Mertens. The nature of computation. Oxford University Press, 2011.

[23] Nicola Santoro, Walter Quattrociocchi, Paola Flocchini, Arnaud Casteigts, and Frédéric Amblard. Time-varying graphs and social network analysis: temporal indicators and metrics. In Proc. of the 3rd Workshop on Social Networks And MultiAgent Systems (SNAMAS), pages 32-38, 2011.

[24] Thomas Schaefer. The complexity of satisfiability problems. In Proc. of the 10th ACM Symposium on Theory of Computing (STOC), pages 216-226, 1978.

[25] Alexander Schrijver. Combinatorial optimization: polyhedra and efficiency, volume 24. Springer Science \& Business Media, 2003.

[26] Yossi Shiloach. Edge-disjoint branching in directed multigraphs. Information Processing Letters, 8(1):24-27, 1979.

[27] Aleksandrs Slivkins. Parameterized tractability of edge-disjoint paths on directed acyclic graphs. SIAM Journal on Discrete Mathematics, 24(1):146-157, 2010.

[28] Robert Tarjan. A good algorithm for edge-disjoint branching. Information Processing Letters, 3(2):51-53, 1974.

[29] Douglas West. Introduction to graph theory, volume 2. Prentice Hall, 2001.

[30] Huanhuan Wu, James Cheng, Silu Huang, Yiping Ke, Yi Lu, and Yanyan Xu. Path problems in temporal graphs. Proc. of the 40th International Conference on Very Large Data Bases (VLDB), 7(9):721-732, 2014. 